\title{
Analysis and Optimization of Supply Chain Traffic using Mobility Mining Techniques
}

\author{
Sabu Augustine \\ Department of Mathematics, Marian College \\ Kuttikkanam, Mahatma Gandhi University \\ Kottayam, Kerala, India
}

\author{
Sajimon Abraham \\ School of Management and Business Studies \\ Mahatma Gandhi University Kottayam, \\ Kerala, India
}

\begin{abstract}
The mobile objects in the supply chain present the means of transportation, and they have an influence on the functioning of the supply chain. The mobile object bring a correct information, where and when necessities, to reduce the uncertainty, increase the visibility of products and increase the global efficiency of the supply chain. The supply chain is a system characterized by the mobility between the various processes of the chain as well as the mobility pattern of materials including the vehicle which carries in transportation network. Mobility mining is the process of extracting hidden knowledge from moving object trajectories. This is a concept paper which visualizes the scope of various mobility mining techniques for analysis and optimization of objects moving in transportation network. Also we demonstrate how the trajectory similarity technique which is one of the mobility mining technique could be used for an efficient and effective supply chain infrastructure.
\end{abstract}

\section{General Terms}

Supply Chain Management, Similarity Measure, Data Mining

\section{Keywords}

Mobile Supply Chain, Optimization, Mobility Mining, Moving Object trajectory, Trajectory Similarity.

\section{INTRODUCTION}

The objective of any company is to produce and to deliver for the precise deadlines, to reduced costs, and satisfy the high levels of service required by the customers. Today the supply chain and its management is an important concept in the companies, which is defines by Mentezer [1] as the systematic, strategic coordination and the tactical management of the actions in the departments of a particular organization, and it aims to improving the performance of every organization.

Mobility is fundamental to economic and social vitality. Economic development and increased mobility have historically progressed in unison, while transportation and investment in its associated infrastructure have become leading indicators of a nation's prosperity. Congestion and and increased mobility has caused serious social concerns: the allocation of scarce land resources for transportation use, the depletion of finite energy resources, and detrimental environmental and safety impacts. In response, governments are imposing limitations on new roadway construction and the automobile itself.

All these challenges requiring for effective management of logistics in supply chain. A planned and efficiently managed transportation network will results in effective movement of goods and services.

\subsection{Mobile Supply Chain}

Mobile SCM (mSCM) refers to the use of mobile applications and devices to aid the conduct of supply chain activities, and ultimately help firms to gain cost reductions, supply chain responsiveness and competitive advantage. The most obvious advantage of using wireless or mobile technology in SCM is that it provides a convenient, time-saving, and highly accurate means of capturing data on movements of goods and other events. These capabilities simplify checking and monitoring tasks and provide up-to-date information on process status. And that's exactly what companies need to create and manage a sophisticated adaptive supply chain network. In addition, mSCM system can be used to streamline business processes of different business functions to ensure efficient flow and exchange of supply chain activities.

\subsection{Mobility Concepts}

Any physical object, that exist in this universe has a basic characteristics that at any point in time, it is located somewhere. In the world that is evolving rapidly, mobility is an important factor of people's life. The Internet, wireless networks, positioning technology as well as personal devices such as PDAs and cell phones and their related services are being advanced and improved day by day. Over the past few years, rapid advances in miniaturization and personalization of electronic devices have taken place, which consequently have resulted in major price reductions. Performance improvements of general computing technologies on the other hand have made it possible to introduce services that previously were even impossible to think of. The ultimate goal of all these advancements is to satisfy the consumer's rising expectations.

People, wildlife, material, food, data and even ideas move in increasing speeds over increasing distances and in increasing volumes. Hence mobility and movement are key processes in our present world. Understanding of mobility patterns is essential to substantiate decision making in public and private sectors, in application domains such as fleet management, transportation modeling, urban planning, tourism, wildlife ecology, spatial epidemiology, location-based services, flight safety, and marine safety. Traffic management can greatly benefit from the analysis of movement data, for example through better movement simulation (leading to better road network designs) but also by incorporating advanced detection sensors in vehicles. It has the following applications in transportation and logistics. 
- Monitoring and planning traffic and public transportation systems

- $\quad$ Localizing new facilities and public services

- Forecasting/simulating traffic-related phenomena

- Geo-marketing and location-based advertising

- Innovative info-mobility services

- Detecting deviations in collective movement behavior.

\section{MOBILITY DATA MANAGEMENT IN TRAJECTORY DATABASES}

A trajectory, the basic form of mobility data, is a sequence of time-stamped locations, sampled from the itinerary of a moving object. Let $\mathrm{T}$ be a set of trajectories in a spatial network, in which each trajectory is represented as

$$
\mathrm{T}=\left(\left(\mathrm{b}_{1}, \mathrm{t}_{1}\right),\left(\mathrm{b}_{2}, \mathrm{t}_{2}\right),\left(\mathrm{b}_{3}, \mathrm{t}_{3}\right), \ldots \ldots \ldots \ldots,\left(\mathrm{b}_{\mathrm{n}}, \mathrm{t}_{\mathrm{n}}\right)\right)
$$

where $\mathrm{n}$ is the trajectory description length, bi denotes a location in binary string and $t_{i}$ is the time instance (expressed in time units, e.g. seconds) that the moving object reached node bi, and $t_{1}<t_{i}<t_{m}$, for each $1<i<m$. It is assumed that moving from a node to another comes at a non-zero cost, since at least a small amount of time will be required for the transition.

A database management system and a data warehouse have been designed around this specific form of movement data. The design of the trajectory database has been influenced by the research on Moving Object Databases (MOD), which extends the traditional database technology for modeling, indexing and querying trajectory data. In MODs, the spatial and temporal dimensions are first-class citizens and both past and current (as well as anticipated future) positions of moving objects are of interest [2][3][4]. Trajectory reconstruction transforms sequences of raw sample points into meaningful trajectories in accordance with different filters: temporal gaps, spatial gaps, maximum speed, tolerance distance, among others.

The different types of queries on trajectory data are

- Spatial (range or nearest-neighbour) search : Find all trajectories that were inside area $\mathrm{A}$ at time instant $\mathrm{t}$ (or time interval I) or Find the trajectory that was closest to point B at time instant $t$ (or time interval I).

- Topological / directional search: Find all trajectories that entered (crossed, left, bypassed, etc.) or were located west (south, etc.) of an area or Find all trajectories that crossed (met, etc.) or were located left of (right of, in front of, etc.) a given trajectory $\mathrm{T}$

- Most-similar-trajectories: Find the $\mathrm{k}$ most similar trajectories to a given trajectory $\mathrm{T}$.

\section{MOBILITY MINING TECHNIQUES}

While the Trajectory Database analytical tools concentrate on presence of moving objects, mobility mining is aimed at analyzing movement. A method for mobility data mining tackles two different tasks: first, to define the format of spatiotemporal patterns and models to be extracted from trajectory data, and second, to design and implement efficient algorithms for extracting such patterns and models. The different mining tasks developed within GeoPKDD [5] focusing on trajectory similarity and patterns, trajectory clustering, and trajectory classification has wider applications in supply chain traffic as explained below.

- By identifying similar trajectories, effective data mining techniques (eg clustering) can be applied to discover useful pattern. For example, a dense cluster is an indication of traffic jam situation which necessitates the expansion of road in future.

- Trajectory similarity can help in several road network applications such as, routing applications which support historical trajectories, logistic applications, city emergency handling, drive guiding systems, flow analysis, etc. In such applications, efficient indexing and query processing techniques are required.

- Knowledge and prediction of the road traffic: Given that the number of vehicles increases on the roads, information related to the density on the network becomes very useful for trip planning.

- Wagon-sharing and Vehicle-to-Vehicle Communication: One of the best way of oil conservation is vehicle sharing which also appears as a solution to traffic congestion in busy roads. Identifying the similar trajectories or even sub-trajectories becomes very useful for such types of applications.

- Transport planning: At the moment of its creation, each road is planned for certain utilization. Reporting trajectory groups allows assessing the suitability of the road infrastructure with its actual use.

The following sections briefly discuss the these mobility mining techniques

\subsection{Trajectory Similarity and Pattern Mining}

Trajectory pattern is a novel notion of spatio-temporal pattern, which formalizes the idea of aggregated movement behaviors. A trajectory pattern, as defined in [6], represents a set of individual trajectories that share the property of visiting the same sequence of places with similar travel times. Therefore, two notions are central: (i) the regions of interest in the given space, and (ii) the typical travel time of moving objects from region to region. In this approach a trajectory pattern is a sequence of spatial regions that, on the basis of the source trajectory data, emerge as frequently visited in the order specified by the sequence; in addition, the transition between two consecutive regions in such a sequence is annotated with a typical travel time that, again, emerges from the input trajectories.

\subsection{Trajectory Clustering.}

Clustering is one of the general approaches to explore and analyze large amounts of data, since it allows the analyst to consider groups of objects rather than individual objects, which are too many. Clustering associates objects in groups (clusters) such that the objects in each group share some properties that do not hold (or hold much less) for the other objects. Spatial clustering builds clusters from objects being spatially close and/or having similar spatial properties (shapes, spatial relationships among components, etc.). Clustering of trajectories implies considering space, time and movement characteristics within a similarity notion: simple distance-based clustering methods are not effective in separating trajectory clusters that exhibit a non convex (non globular) shape, as it often occurs in practice 


\subsection{Trajectory Classification and Location Prediction}

Predictive models for trajectory data include a classification method for inferring the category of a trajectory, (e.g., the transportation means associated to a trajectory: private car, public transportation, pedestrian, etc.), and a predictor of the next location of a moving object given its past trajectory. There is strong current interest in next location prediction, in that it enables several intelligent location-based services. In the literature, this task is achieved by applying various learning methods to the history of each moving object for the purpose of creating an individual location predictor.

\subsection{Trajectory Anonymity}

In the context of personal mobility data, privacy is a big concern: location data allow inferences which may help an attacker to discover private information, such as individual habits and preferences. Hiding explicit identifiers and replacing them with pseudonyms is insufficient to guarantee anonymity, since location represents a property that may allow re-identification: for instance, characteristic locations such as home and work place can be easily uncovered with the use of visual analytics methods, given detailed personal trajectories. Therefore, in all cases when privacy concerns are relevant, the trajectory data cannot be disclosed without appropriate safeguards. Anonymization techniques are data transformations that aim at a double goal: decrease the probability of re-identification below an acceptable threshold, while at the same time maintaining the analytical utility of the data.

\subsection{Visual Analytics}

The aim of this system is to help the analyst to navigate through mobility data and patterns and to visually drive the analytical process [7]. The key features include: the visualization of Trajectory patterns to support the navigation of the extracted patterns in the spatial and temporal dimensions; the progressive refinement of Trajectory clusters, through user-driven exploration and evaluation of the discovered Trajectory clusters [8] and the visual exploration of various measures provided by the Trajectory Warehouse [9].

\section{ANALYSIS OF TRAFFIC MOVEMENTS IN SUPPLY CHAIN}

Here we concentrate our study on spatio-temporal similarity and pattern mining of mobility data.

\section{Spatial Similarity Matrix based on POI}

We introduce the similarity measures between two moving object trajectories in a constrained network incorporating concepts discussed in [10]. Here the spatial similarity is measured based on three concepts as explained below.

a) Common locations visited by two trajectories

b) Structural similarity of locations in the trajectories

c) Sequence Similarity of trajectories

d) Temporal refinement of trajectories

\section{a) Common locations visited by two trajectories}

Let $\mathrm{Ti}$ and $\mathrm{Tj}$ be two trajectories. We introduce a Spatial Similarity measure $\operatorname{Sim}\left(\mathrm{T}_{\mathrm{i}}, \mathrm{T}_{\mathrm{j}}\right)$ which attempt to incorporates number of common locations in trajectories. This similarity matrix measures the number of common locations passed through during the two trajectories relative to the total number of locations in both trajectories.

\author{
$\operatorname{Sim}\left(T_{i}, T_{j} ; P\right)=1$ if $\forall p$ in $P$; $p$ is on $T_{i}$ and $T_{j}$ \\ 0 ; otherwise \\ Where $\mathrm{P}$ is the given set of Points of Interest
}

\section{b) Structural Similarity}

Here structural similarity we mean how geographically the points are closed to each other. In order to measure Structural similarity we consider each of the four strings, separately and for each bit string, weightage will be assigned from left to right in the order $1,2,3$ etc. Then we compare each corresponding bit of the two token strings bit by bit from the beginning until the first pair of bits is different. The similarity between two token strings is defined as the sum of the weight of those matching tokens divided by the sum of the total weights.

\section{c. Sequence Similarity of web sessions}

The above spatial similarity measures consider the number common locations visited by two trajectories and their geographical neighborhood as a measure of structural similarity. The spatial similarity is checking only the percentage of common locations visited by each input trajectory with the query trajectory made by points of interest. As we consider important individual locations in Point of interest(POI), the sequence in which these locations visited by an input trajectory is also to be considered in finding the actual similarity.

For example in the field of supply chain transport if POI contains set of outlets for supply and then the sequence of locations a wagan crosses will also have to be considered in finding how a user movement trajectory matches with the query trajectory created with the given set of POI. Here we consider the original trajectory data as a set of sequences, and apply sequence alignment method to measure similarity between trajectories. The sequencing is being done by the dynamic programming technique.

\section{d Temporal Refinement}

The similarity measure defined in the previous section takes into consideration only the spatial concept, which consists of structural similarity and sequence similarity. In real applications, the time information associated with each trajectory is also very important. So to measure the similarity we have to consider the concept of space and time together. Here we are considering the temporal distance by taking the time differences in visiting each location as shown in Fig 1.

Temporal distance between Trajectory A $\left(\mathrm{T}_{\mathrm{A}}\right)$ and Trajectory $\mathrm{B}\left(\mathrm{T}_{\mathrm{B}}\right)$ will be

$$
\begin{aligned}
\operatorname{dist}_{\mathrm{T}}\left(\mathrm{T}_{\mathrm{A}}, \mathrm{T}_{\mathrm{B}}\right)= & \text { Differences in time at common } \\
& \text { locations visited -P1,P3,P5 } \\
& =10+5+0=15
\end{aligned}
$$

\section{EXPERIMENTAL EVALUATION \& RESULTS}

We have taken a real-world data of traffic movement for experimentation purposes, namely a fleet of shipment wagons that make trajectory data set. The data set consists of 576 trajectories of 150 wagons moving places around Athens metropolitan area in Greece for 30 distinct days. The structure of each record is as follows: 


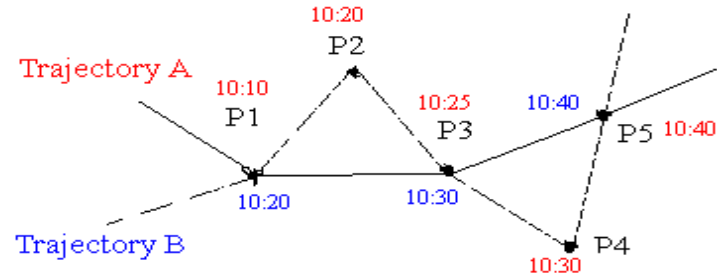

Fig 1: Temporal Distance of Two Trajectories

\{obj-id, traj-id, date(dd/mm/yyyy), time(hh:mm:ss), lat, lon, $\mathrm{x}, \mathrm{y}\}$, where (lat, lon) is in WGS84 reference system and (x,y) is in GGRS87 reference system.

All experiments have been conducted on a Intel Core 2 Duo machine running Windows XP, with 2 GB of RAM, and a 320 GB SATA2-16 MB hard disk using the visual Programming Language Visual Basic. Each of the three methods generate

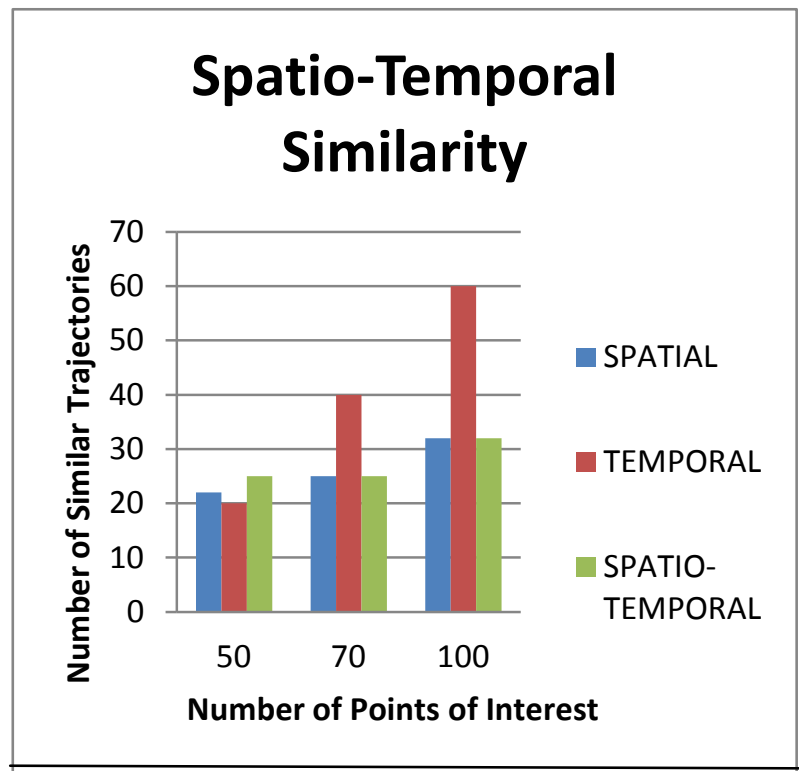

Fig 2. Comparison of Spatial, Temporal and Spatio-Temporal Similarity Search

knowledge based on the methods proposed which can be used for mining tourist's movement profiles. The results of experiments confirm that (Fig 2) the spatio-temporal similarity search is more consistent than other two types of similarity search.

\section{CONCLUSION}

The similarity problem in trajectory database for moving objects on road networks has many applications in
Transportation Network like identification of traffic congestion and re-routing etc. In this paper we have proposed a method to measure the spatio-temporal similarity of moving object trajectories highlighting applications in mining mobility data. We have visualized other mobility mining techniques that has wider applications in optimizing supply chain traffic. As a continuation work we are planning to use mathematical programming tools to further optimize these process to have a cost efficient supply chain management system.

\section{REFERENCES}

[1] Mentzer, J.,T.2000. Supply Chain Management. Library of congress cataloging in publication data.

[2] R.H. Guting,, SECONDO: 2005. An Extensible DBMS Platform for Research Prototyping and Teaching. In Proceeding of the International Conference on Data Engineering, ICDE, pages 1115-1116, Tokyo, Japan.

[3] M.F. Mokbel, 2004. PLACE: A Query Processor for Handling Real-time Spatio-temporal Data Streams (Demo). In Proceeding of the International Conference on Very Large Data Bases, VLDB, pages 1377-1380, Toronto, Canada.

[4] O. Wolfson, 2000. Management of Dynamic Location Information in DOMINO (Demo). In Proceeding of the International Conference on Extending Database Technology, EDBT, pages: 769-771.

[5] Fosca Giannotti, Roberto Trasarti , 2010. Mobility, Data Mining and Privacy: The GeoPKDD Paradigm, KDD Lab -ISTI - CNR Pisa, Italy \& Center for Complex Network Research, Northeastern University, Boston.

[6] F. Giannotti, M. Nanni, F. Pinelli, and D. Pedreschi. 2007. Trajectory pattern mining. In KDD, pages 330 339.

[7] G.L. Andrienko, N.V. Andrienko, 2008. A Visual Analytics Approach to Exploration of Large Amounts of Movement Data.VISUAL pages 1-4

[8] S. Rinzivillo, D. Pedreschi, M. Nanni, F. Giannotti, N. Andrienko, and G. Andrienko. 2008. Visually-driven analysis of movement data by progressive clustering. Information Visualization, pages 225-239. .

[9] S. Orlando, 2007. Spatio-Temporal Aggregations in Trajectory Data Warehouses. Proceedings of DaWaK.

[10] Sajimon Abraham, Sojan Lal P., 2010. Trajectory similarity of Network Constrained Moving Objects and Applications to Traffic Security, Pacific Asia International Workshop On Security Informatics(PAISI 2010) held in 21 June , Hyderabad , India, LNCS 6122, pp. 31-43, Springer-Verlag Berlin Heidelberg. 